\title{
IMBEDDINGS, IMMERSIONS, AND CHARACTERISTIC CLASSES OF DIFFERENTIABLE MANIFOLDS
}

\author{
STAVROS PAPASTAVRIDIS
}

\begin{abstract}
Let $I_{n}^{i}$ be the set of mod-2 characteristic classes which are of dimension $i$, and they are zero for all $n$-dimensional smooth manifolds. Let $I_{n, k}^{i}$ be the set of $i$-dimensional mod-2 characteristic classes which are zero for all $\boldsymbol{n}$-dimensional smooth manifolds which immerse in codimension $\boldsymbol{k}$, (we are talking about normal characteristic classes). Let $K$ be the (graded) ideal in $H^{*}\left(B O, Z_{2}\right)$ generated by $w_{k+1}, w_{k+2}, \ldots$ Then if $i<(n+k) / 2$, we have $I_{n, k}^{i}=I_{n}^{i}+K^{i}$. We have some related results for imbedded manifolds, and also for manifolds which immerse or imbed with an $S O, U, S U$, Spin, etc. structure on the normal bundle.
\end{abstract}

Introduction. Let $f_{r}: X_{r} \rightarrow B O(r)$ be a sequence of fibrations with maps $g_{r}$ : $X_{r} \rightarrow X_{r+1}$ such that the usual diagrams commute. For such a situation Lashof defines the concept of an $X$-structure on manifolds [7], and proves a Thom-isomorphism for the bordism groups of such manifolds. Many of the usual classes of manifolds may be described in terms of $X$-structures, e.g. $U, S O$, Spin, etc., as well as some more esoteric classes of manifolds.

In this paper we study $X$-characteristic classes mod-p. i.e. the group $H^{*}(X)=$ proj $\lim H^{*}\left(X_{r} ; Z_{p}\right)$. In particular we are interested in those characteristic classes which go to zero by the normal map of all $n$-manifolds which imbeds or immerses in codimension $k$, (by normal map I mean the lift of the Gauss map $M \rightarrow B O(r))$.

Let us assume that the map $g_{r}^{*}: H^{*}\left(X_{r+1} ; Z_{p}\right) \rightarrow H^{*}\left(X_{r} ; Z_{p}\right)$ is an isomorphism in dimensions not greater than $r$. Furthermore we assume that the pull-back over $X_{r}$ of the universal $r$-linear bundle, $\gamma_{r}$, is $Z_{p}$-orientable. Throughout this paper $n, k, p$ are fixed.

THEOREM 1. An X-characteristic class of dimension less than $k$, is zero on all $n$-manifolds which imbed in codimension $k$ with an $X$-structure on its normal bundle, if and only if it is zero on all n-manifolds which have an $X$-structure.

Let $I_{n}$ be the graded set of all $X$-characteristic classes which are zero on all $n$-manifolds with an $X$-structure, and $I_{n, k}$ be the graded set of all $X$ characteristic classes which are zero on all $n$-manifolds which immerse in codimension $k$ with an $X$-structure on its normal bundle. Let $l_{r}: H^{*}(X$; $\left.Z_{p}\right) \rightarrow H^{*}\left(X_{r} ; Z_{p}\right)$ be the obvious map.

Received by the editors April 12, 1977.

AMS (MOS) subject classifications (1970). Primary 55F40, 57D40.

$\mathrm{Key}$ words and phrases. Immersions, imbeddings, characteristic classes.

๑ American Mathematical Society 1978 
THEOREM 2. If $i \leqslant(n+k) / 2$, then $I_{n, k}^{i}=I_{n}^{i}+\left(\operatorname{ker} l_{k}\right)^{i}$.

The theorem above generalizes and extends the results of [1]. $M$. Bendersky proves in (1), that $I_{n, k}^{i}=I_{n}^{i}$ if $i \leqslant k$, for certain $X$-structures.

What happens in higher dimensions, is an interesting and difficult question.

1. The case of immersed manifolds. From now on we adopt the following notational conventions. All cohomology groups will have $Z_{p}$ coefficients. $K$ will be $K\left(Z_{p}, n-i\right)$, where $i$ is a natural number not greater than $n$. We put $K_{+}=K U$ \{point , and let $c$ be the fundamental class of $K$. If $G$ is a group then $G^{*}=\operatorname{Hom}\left(G, Z_{p}\right)$. We select Thom classes $U_{r} \in H^{r}\left(T\left(\gamma_{r}\right)\right)$ such that if $T g_{r}$ is the obvious map $T\left(\gamma_{r}+\varepsilon\right) \rightarrow T\left(\gamma_{r+1}\right),(\varepsilon$ is the trivial one-dimensional bundle), then $\operatorname{Tg}_{r}^{*}\left(U_{r+1}\right)=S U_{r}$, (the letter $S$ denotes the suspension). $N$ will be a natural number very big in comparison with $n$ and $k$. We put $A^{\prime}=$ $S^{N-k} T \gamma_{k} \wedge K_{+}, A=S^{N-k} T \gamma_{k} \wedge K, B^{\prime}=T \gamma_{N} \wedge K_{+}$, and $B=T \gamma_{N} \wedge K$.

LEMMA 1.1. The maps $\pi_{j}^{*}(A) \rightarrow \pi_{j}^{*}\left(A^{\prime}\right)$ and $\pi_{j}^{*}(B) \rightarrow \pi_{j}^{*}\left(B^{\prime}\right)$ induced respectively by the obvious maps $A^{\prime} \rightarrow A$ and $B^{\prime} \rightarrow B$, are monomorphisms in the stable range.

Proof. We will prove only the first case, the other one being similar. We observe that the spaces $A, A^{\prime}$ are highly connected. The obvious projections $A^{\prime} \rightarrow A$ and $A^{\prime} \rightarrow S^{N-k} T \gamma_{k}$ induce the product map $A^{\prime} \rightarrow A \times S^{N-k} T \gamma_{k}$ which is an iso in $Z_{p}$-cohomology in the stable range so it is an iso in $Z_{p}$-homotopy. The obvious projection $A \times S^{N-k} T \gamma_{k} \rightarrow A$ is onto in homotopy, so the composition

$$
A^{\prime} \rightarrow A \times S^{N-k} T \gamma_{k} \rightarrow A,
$$

is epi in $Z_{p}$-homotopy in the stable range. So the dual map $\pi^{*}(A) \rightarrow \pi^{*}\left(A^{\prime}\right)$ is mono in the stable range.

Let us consider the cofibration $S^{N-k} T \gamma_{k} \rightarrow T \gamma_{N} \rightarrow L$.

LEMMA 1.2. The space $L \wedge K$ is a product of $K\left(Z_{p}\right.$, *)'s in $Z_{p}$-cohomology up to dimension $N+k+1+2(n-i)$.

Proof. From the cohomology exact sequence of the above cofibration we get that the space $L$ is $(N+k)$-connected because the map $S^{N-k} T \gamma_{k} \rightarrow T \gamma_{N}$ induces an iso in $Z_{p}$-cohomology up to dimension $N+k$. Since $H^{*}(K)$ is free $A_{p}$-module up to dimension $2(n-i)$, then $H^{*}(L \wedge K)$ is a free $A_{p}$-module up to dimension $N+k+1+2(n-i)$. And the result follows.

From the very elementary homological algebra we borrow the following

LEMMA 1.3. Let $X \rightarrow Y \rightarrow Z$ be an exact sequence of abelian groups such that $Z$ is a direct sum of $Z_{p}$ 's. Then the dual sequence $Z^{*} \rightarrow Y^{*} \rightarrow X^{*}$ is exact.

Proof. It comes down to the fact that the image of the map $Y \rightarrow Z$ is a direct summand of $Z$.

And now we are ready to prove Theorem 2.

Proof of Theorem 2. We define the map $G^{\prime}: H^{i}\left(X_{N}\right) \rightarrow \pi_{N+n}^{*}\left(B^{\prime}\right)$ by the formula $G^{\prime}(x)([a])=a^{*}\left(x U_{N} \wedge c\right)$, where $x$ is an element of $H^{i}\left(X_{N}\right)$ and $[a]$ 
is an element of $\pi_{N+n}\left(B^{\prime}\right)$. By (2), $I_{N}^{i}$ is the kernel of $G^{\prime}$. In the same way we define the map $G: H^{i}\left(X_{N}\right) \rightarrow \pi_{N+n}^{*}(B)$ and we get the commutative diagram

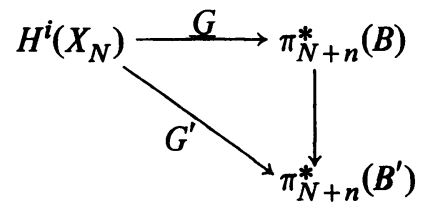

where the vertical map is mono by Lemma 1.1. So $I_{N}^{i}$ is the kernel of $G$.

In exactly the same way we define maps $F^{\prime}: H^{i}\left(X_{k}\right) \rightarrow \pi_{N+n}^{*}\left(A^{\prime}\right)$, by

$$
F^{\prime}(x)([a])=a^{*}\left(x S^{N-k} U_{k} \wedge c\right),
$$

and the map $F: H^{i}\left(X_{k}\right) \rightarrow \pi_{N+n}^{*}(A)$ defined the same. By $(10), I_{n, k}^{i}$ is the kernel of $G^{\prime}$ and because of Lemma 1.1, it is the kernel of $G$. We consider the cofibration $A \rightarrow B \rightarrow L \wedge K$ which comes from the cofibration $S^{N-k} T \gamma_{k} \rightarrow$ $T \gamma_{N} \rightarrow L$ by smashing with $K$. Since we are able in the stable range this cofibration is a fibration too, so it gives exact sequences in homotopy and cohomology. We have the commutative diagram

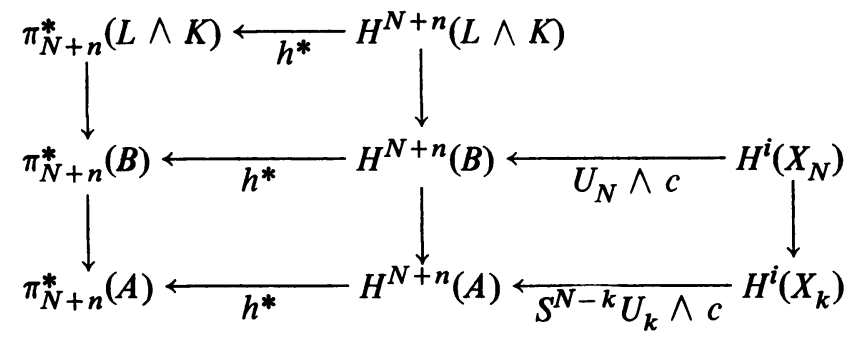

where the first and second column is the dual of the homotopy and homology exact sequence respectively, of the cofibration. They are exact by Lemmas 1.2 and 1.3 , since $i \leqslant(n+k) / 2$. The third column is essentially $l_{k}$. The horizontal maps $h^{*}$ are the dual of the mod-p Hurewicz homomorphism of the corresponding space, and the first one is epi because of Lemma 1.2. The composition of maps in the second row gives the map $G$, and in the third row the map $F$.

The result follows by chasing around the diagram.

2. The case of imbedded manifolds. The proof of Theorem 1 , follows the same lines like Theorem 2.

Analogously with $A^{\prime}, A, B^{\prime}, B$ we put $C^{\prime}=T \gamma_{k} \wedge K_{+}, C=T \gamma_{k} \wedge K$, $D^{\prime}=\Omega^{N-k} T \gamma_{N} \wedge K_{+}$, and $D=\Omega^{N-k} T \gamma_{N} \wedge K$.

LEMMA 2.1. The maps $\pi_{j}^{*}(C) \rightarrow \pi_{j}^{*}\left(C^{\prime}\right), \pi_{j}^{*}(D) \rightarrow \pi_{j}^{*}\left(D^{\prime}\right)$, induced respectively by the obvious maps $C^{\prime} \rightarrow C$ and $D^{\prime} \rightarrow D$, are mono if $j$ is less than $k+(n-i)+k$.

Proof. Like Lemma 1.1. 
Proof of Theorem 1. In a way analogous with the definition of $G^{\prime}, G, F^{\prime}, F$ in the previous section, we define maps

$$
R^{\prime}: H^{i}\left(X_{N}\right) \rightarrow \pi_{n+k}^{*}\left(D^{\prime}\right)
$$

by

$$
\begin{gathered}
R^{\prime}(x)([a])=a^{*}\left(\Omega^{N-k}\left(x U_{N}\right) \wedge c\right) \\
R: H^{i}\left(X_{N}\right) \rightarrow \pi_{n+k}^{*}(D), P^{\prime}: H^{i}\left(X_{k}\right) \rightarrow \pi_{n+k}^{*}\left(C^{\prime}\right)
\end{gathered}
$$

by

$$
P^{\prime}(x)([a])=a^{*}\left(x U_{k} \wedge c\right) \text { and } P: H^{i}\left(X_{k}\right) \rightarrow \pi_{n+k}^{*}(C) .
$$

Again $I_{n}^{i}$ is the kernel of $R$, and the set of $X$-characteristic classes which are zero on all $n$-manifolds with an $X$-structure which imbeds in $R^{n+k}$ is the kernel of $P$, if $i \leqslant k-1$. On the other hand the obvious map $C \rightarrow D$ induces iso in cohomology up to dimension $(2 k+n-i)=1$, so we get a monomorphism up to dimension $(2 k+n-i-1)$ in the map $\pi^{*}(D) \rightarrow$ $\pi^{*}(C)$, and the theorem follows.

\section{REFERENCES}

1. M. Bendersky, Characteristic classes of $n$-manifolds immersing in $R^{n+k}$, Math. Scand. 31 (1972), 293-300.

2. E. H. Brown and F. P. Peterson, Relations among characteristic classes. I, Topology 3 (1964), 39-52.

3. E. H. Brown and F. P. Peterson, Relations among characteristic classes. II, Ann. of Math. (2) 81 (1965), 356-363.

4. R. L. Brown, Imbeddings, immersions, and cobordism of differentiable manifolds, Bull. Amer. Math. Soc. 76 (1970), 763-766.

5. H. Cartan, Sur les groupes d'Eilenberg-Mac Lane. II, Proc. Nat. Acad. Sci. U.S.A. 40 (1954), 704-707.

6. M. Hirsch, Immersions of manifolds, Trans. Amer. Math. Soc. 93 (1959), 242-276.

7. R. Lashof, Poincaré duality and cobordism, Trans. Amer. Math. Soc. 109 (1963), 257-277.

8. R. Stong, Notes on cobordism theory, Princeton Univ. Press, Princeton, N. J., 1968.

9. R. Thom, Quelques propriétés globales des variétés différentiables, Comm. Math. Helv. 28 (1954), 17-86.

10. R. Wells, Corbordism of immersions, Topology 5 (1966), 281-294.

Mathematical Institute, University of Athens, Athens 143, Greece

Current address: Department of Mathematics, University of Crete, Iraklion, Crete, Greece 